\title{
“A Withered Limb”: Vanishing bone disease (Gorham's disease) of the upper limb
}

K.A.M.S. Jayachandra, ${ }^{1}$ A De Silva, ${ }^{2}$ G.L Punchihewa, ${ }^{2}$ S. Sutharsan ${ }^{2}$

${ }^{1}$ Deparment of Radiology, National Hospital of Sri Lanka

${ }^{2}$ Department of Orthopaedic and Trauma Surgery, National Hospital of Sri Lanka

\section{Abstract}

Vanishing bone disease (VBD) is a rare bone disorder of unknown etiology. Histologically it is characterized by non-malignant proliferation of epithelial lined vascular and lymphatic channels and increased number of osteoclasts that result in resorption of osseous matrix. Lost bone is replaced by granulation tissue. The clinical presentation of VBD is variable and depends on the site of involvement.

Key Words: Vanishing Bone Disease

Copyright:

Correspondence: K.A.M.S. Jayachandra 


\section{Introduction}

The first known reported case of this rare disease was in 1838 by JBS Jackson in The Boston Medical and Surgical Journal (now The New England Journal of Medicine). ${ }^{1}$ In 1954 Gorham and Stout presented an overview of 24 cases reported at that time which led to the disease also being called Gorham - Stout syndrome or Gorham's disease. ${ }^{2}$ Out of approximately 200 cases reported in the literature ${ }^{3}$, single bone involvement is common though multicentric involvement crossing joints is also reported..$^{3-5}$ Even rarer is multicentric involvement crossing multiple joints, with only a single case documented so far. ${ }^{7}$ We report a case of multicentric VBS crossing glenohumoral, arcomioclavicular and elbow joints, which to our knowledge is the second reported case of VBS crossing multiple joints of upper limb as well as the case involving highest number of joints.

\section{Case Report}

A 49year old previously healthy female presented with right upper limb pain for 8 weeks duration. Pain was not radiating and not localized. She gave a history of minor trauma with a fracture of proximal $1 / 3$ of right humerus 6 months back which was managed conservatively. On examination her shoulder movements were severely restricted and the upper limb was oedematous and flaccid. No skin changes were seen. She was afebrile. Her past medical history was unremarkable.

Plain radiographs revealed massive osteolysis across glenohumoral articulation, involving whole of right humerus except distal articular surface, glenoid process of right scapula, lateral $1 / 3$ of clavicle and acromion process of scapula (Fig.1).

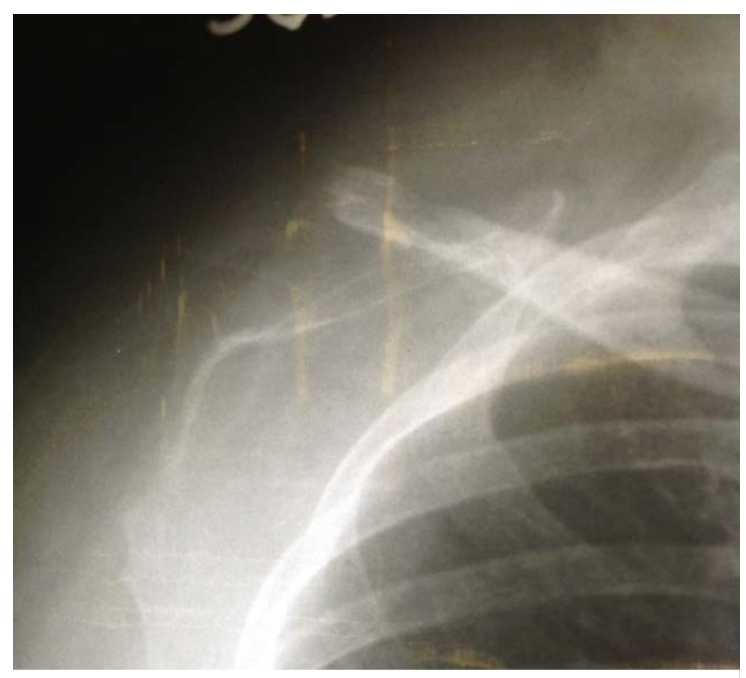

Figure 1: AP radiograph of right shoulder showing extensive osteolysis of $\mathrm{R} /$ humerus, lateral $1 / 3^{\text {rd }}$ of $\mathrm{R} /$ clavicle, glenoid process and acromion process of R/scapula

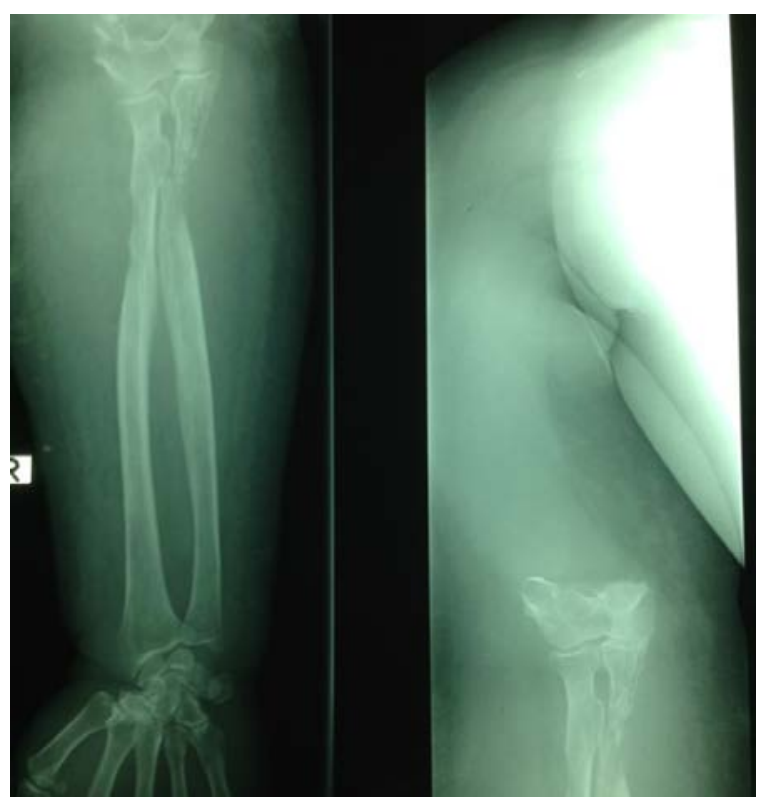

Figure 2: AP radiographs showing extensive osteolysis spreading across the joints involving right humerus, outer $1 / 3$ of clavicle, glenoid cavity and proximal $1 / 3$ of ulna. 

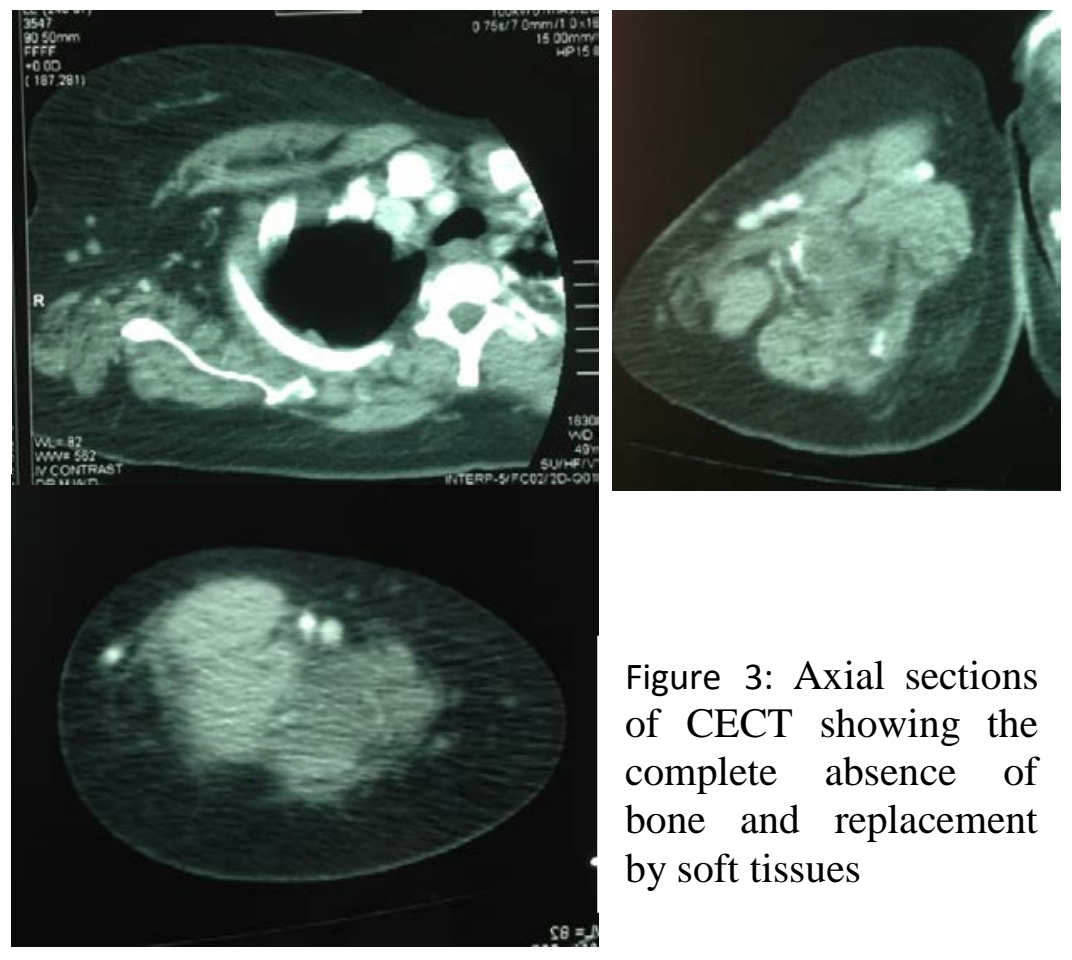

Figure 3: Axial sections of CECT showing the complete absence of bone and replacement by soft tissues

Histology of open true cut biopsy showed no malignant cells, but revealed thinned out bone trabeculae and marrow space expansions replaced by abundant proliferating vascular channels (Fig.5).

\section{Discussion}

VBD is a nonhereditary disease of unknown aetiopathogenesis. No gender predilection is demonstrated and young adults are generally affected. Predominant histopathology is bone resorption, the aetiology of which is still controversial. Gorham et al. postulated progressive angiomatous replacement of bone with increased activity of osteoclasts due to chemical changes in tissues. ${ }^{8}$ Since 1980 s attention is focused on cytochemical aspect of the disease which unravelled a possible role of interleukin-6 (IL-6) in disease causation. $^{9}$

proximal right radius and ulna also showed patchy bone destruction sparring articular surfaces (Fig.2). Rest of the skeleton was normal in the skeletal survey. NCCT of right shoulder (Figure 3) revealed absence of bone in mid and proximal arm. As the patient had generalized swelling of right upper limb, a duplex study was performed in order to exclude a vascular cause for oedema. It revealed multiple abnormal hypoechoeic vascular channels which displayed flow in the power doppler mode only. This was followed by CT angiography (Figure 4) which revealed normal deep arterial and venous systems.

Heamatological investigations and biochemical profile including serum Alkaline phosphatase levels were all normal.

Insidious onset of clinical symptoms of nonspecific limb pain as in our case, limb weakness and impaired function, swelling and deformity are reported. Patients may present with acute pain due to pathological fractures. Disease may develop in any region of the skeleton, but great majority of reported cases involve maxillofacial region and upper limb including scapula. ${ }^{3}$ Diagnosis is based on clinical, radiological and histological evidence. Since there is no biochemical derangement, laboratory tests are often negative. Most dramatic changes of VBD are seen in plain radiographs. Subcortical and intramedullary lucencies followed by progressive fragmentation and ultimate dissolution was described by Resnik. ${ }^{10}$ Ends of the remaining bone appear pointed. The disease process may involve 


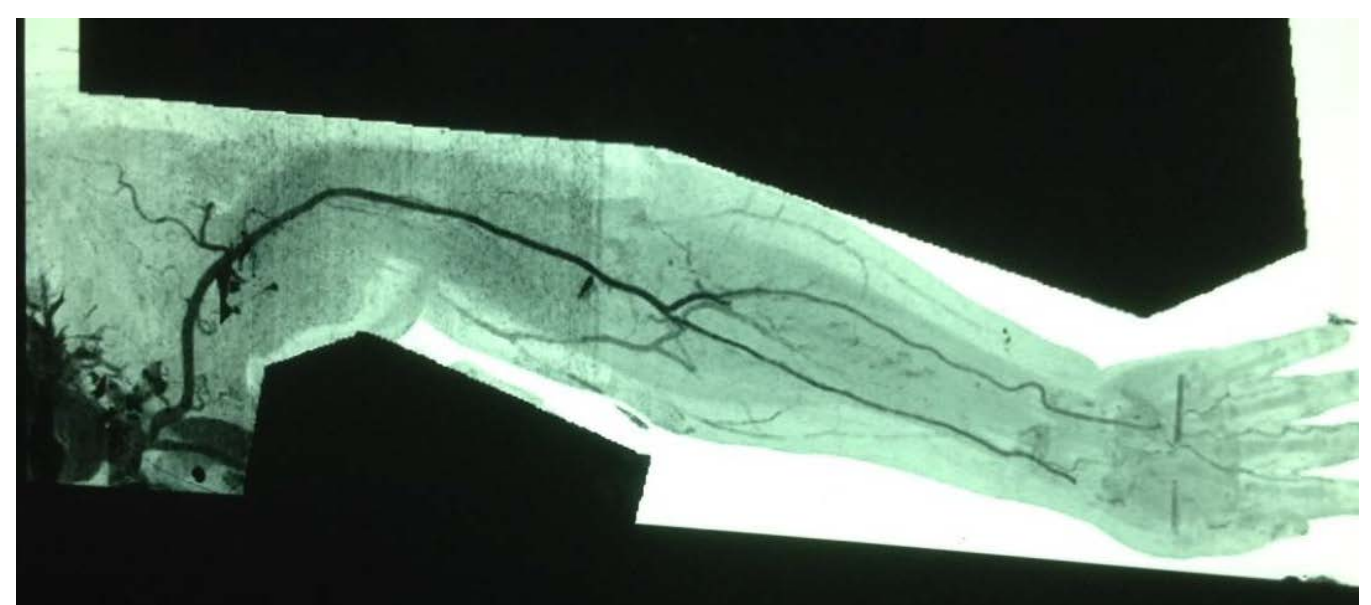

Figure 4:

CT angiogram showing normal right upper limb vessels
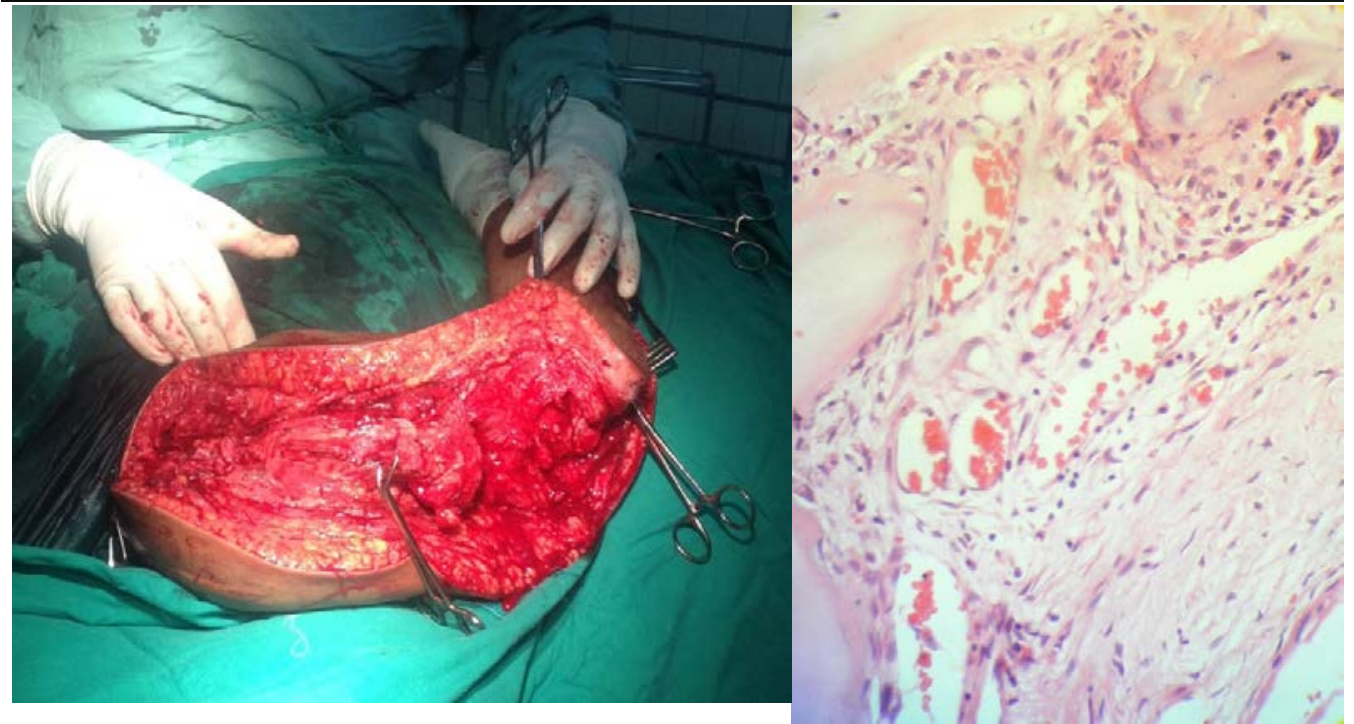

Figure 5:

Macroscopic and microscopic appearance of multiple vascular channels within the fibrous tissue replacing the bone

contiguous bones across joints as in our case. Such patterns of regional osseous destruction should alert the radiologists to the diagnosis. Radioisotope studies and MRI are only supplementary and yield nonspecific findings.

Although VBD is a benign entity the prognosis is unpredictable but definitely disabling. The osseous destruction progresses unabated over several years and may eventually stabilize spontaneously or regress. ${ }^{11}$ Involvement of ribs and vertebra can cause intrathoracic complications such as chylothorax.
Above mentioned clinical, radiological and histological features make VBD distinguishable from many local and systemic conditions which are associated with bone resorption such as disuse atrophy, Sudeck's atrophy, endocrine diseases, primary and metastatic carcinoma etc. Since the disease aetiology is still in the dark no standard therapy is yet available. Currently bisphosphonates are used to suppress osteoclasts and alpha-2b interferon for antiangiogenesis and prevention of interleukin-6 production. ${ }^{12}$ Radiotherapy has shown to be helpful in curbing the disease progression and reducing complications. ${ }^{12}$ 


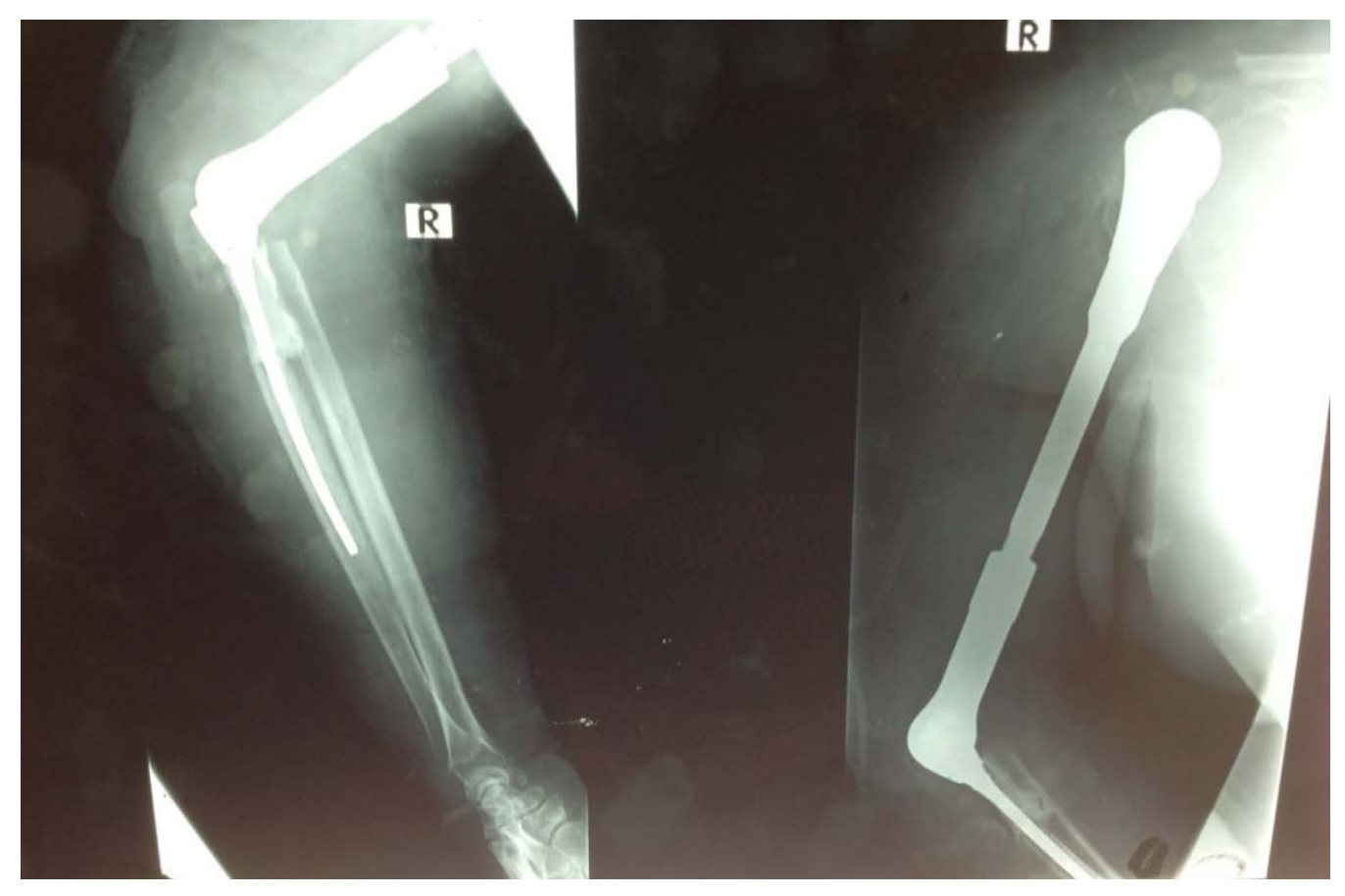

Figure 6: Post operative $\mathrm{x}$ ray with the custom made endoprosthesis

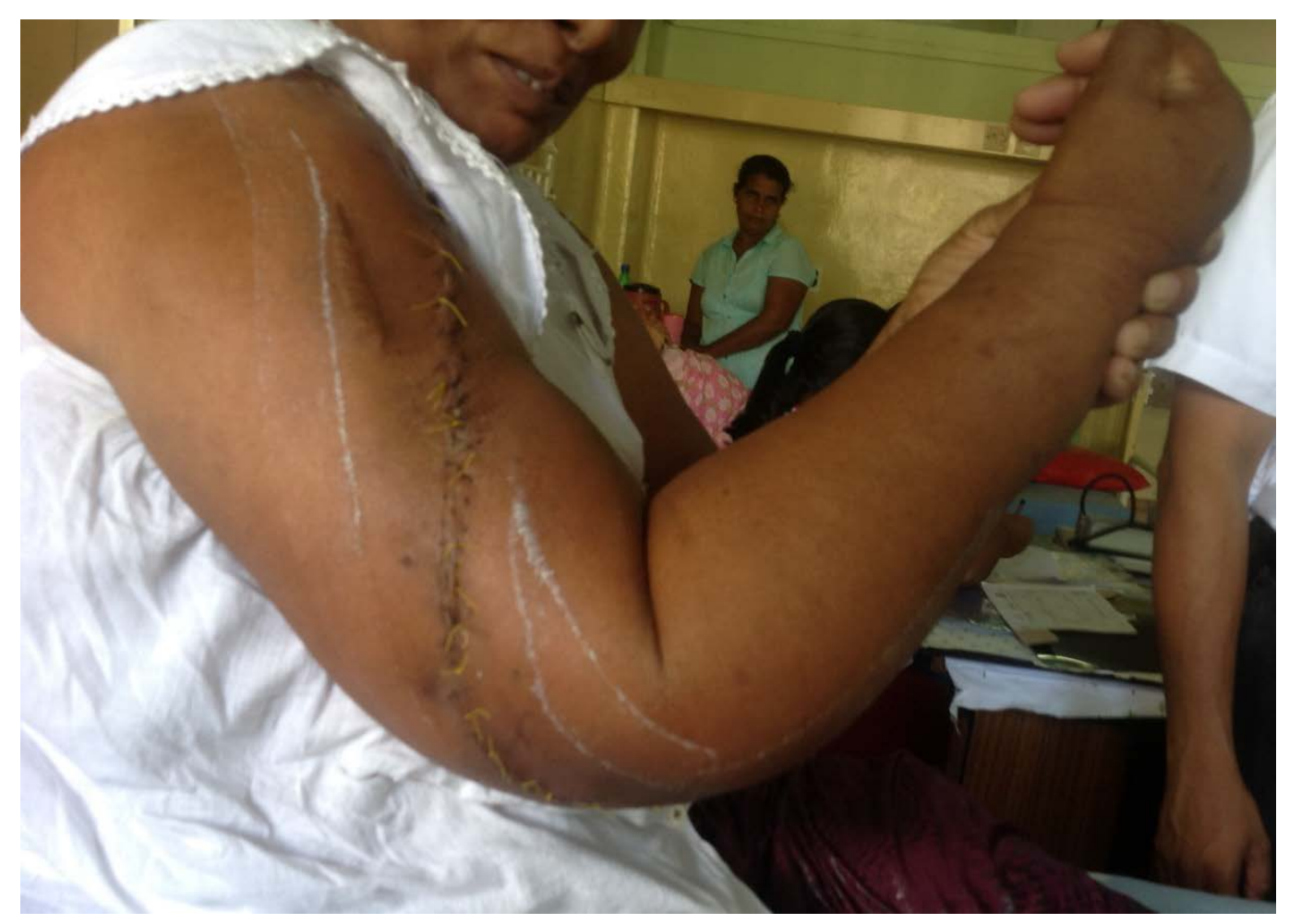

Figure 7: R/ upper limb of the patient after the surgery (taken with informed consent of patient) 
Surgical options include resection of the lesion, and reconstruction using bone grafts and/or prostheses. This option is more suited for patients with monostatic disease. However, our patient with multicentric disease crossing multiple joints, underwent right upper limb reconstruction surgery with custom made titanium mega prosthesis for the humerus and proximal ulna with reconstruction of R/elbow joint (Figs 6 \& 7).

\section{Summary}

VBD is a rare disease of unknown aetiology with remarkable plain radiographic appearances overlapping with much commoner entities such as malignancy, infection, and endocrine disorders. Radiologist with a high index of suspicion may be the first to suggest the diagnosis enabling early intervention before severe functional disability develops.

\section{References:}

1. Jackson JBS. A boneless arm. Boston Med Surg J 1838;18:368-9.

2. Gorham LW, Stout AP. Massive osteolysis (acute spontaneous absorption of bone, phantom bone, disappearing bone): its relation to hemangio-matosis. Journal of Bone and Joint Surgery. American.1955;37:985-1004.

3. S.A. Papadakis, L. Khaldi, E. C. Babourda, S. Papadakis, T. Mitsitsikas, and G. Sapkas, Vanishing bone disease: review and case reports, Orthopedics, Vol. 31, No. 3, p. 278, 2008.

4. L. Scialpi, M. Servedio, B. Moretti, M. E. Solarino, D. Guglielmo, and G. Solarino, Gorham's disease: a rare case of multicentric localization, La Chirurgia Degli Organi di Movimento, Vol. 89, No. 4, pp. 339-345, 2004.
5. Tauro B, Multicentric Gorham's disease, Journal of Bone and Joint Surgery. British, Vol. 74, No. 6, pp. 928-929, 1992.

6. T. Tyler and H. D. Rosenbaum, Idiopathic multicentric osteolysis, American Journal of Roentgenology, Vol. 126, No. 1, pp. 23-31, 1976.

7. Elke R. Ahlmann, Yanling Ma, Vonny Tunru Dinh. A rare case report of extensive polyostotic Gorhams disappearing bone disease involving the upper extremity. Case rep Orthop. 2011; 2011: 486756. http://dx.doi.org/10.1155/2011/486756

8. L. W. Gorham and A. P. Stout, Massive osteolysis (acute spontaneous absorption of bone, phantom bone, disappearing bone): its relation to hemangiomatosis, Journal of Bone and Joint Surgery. American, Vol. 37, pp. 9851004, 1955.

9. R. D. Devlin, H. G. Bone, and G. D. Roodman, Interleukin-6: a potential mediator of the massive osteolysis in patients with GorhamStout disease, Journal of Clinical Endocrinology and Metabolism, Vol. 81, no. 5, pp. 1893-1897, 1996.

10. Resnick D. Chapter 89. Osteolysis and chondrolysis. In: Resnick D, ed. Diagnosis of Bone and Joint Disorders. Fourth Edition. Philadelphia, Pennsylvania, U.S.A.: W.B. Saunders Company; 2002. Volume 5. pages 4920-4944

11. S. R. Cannon, "Massive osteolysis. A review of seven cases,” Journal of Bone and Joint Surgery. British, vol. 68, no. 1, pp. 24-28, 1986.

12. Hagberg H, Lamberg K, Astrom G. Alpha-2b interferon and oral clodronate for Gorham's disease. Lancet 1997;350 (9094):1822-1823.

13. S. F. Dunbar, A. Rosenberg, H. Mankin, D. Rosenthal, and H. D. Suit, Gorham's massive osteolysis: the role of radiation therapy and a review of the literature, International Journal of Radiation Oncology Biology Physics, vol. 26, no. 3, pp. 491-497, 1993. 